\section{Magnesium-Gabe senkt den Blutdruck von Patienten mit gestörtem Stoffwechsel}

Dibaba DT et al. The effect of magnesium supplementation on blood pressure in individuals with insulin resistance, prediabetes, or noncommunicable chronic diseases: a meta-analysis of randomized controlled trials. Am J Clin Nutr 2017; 106: 921-929

Maßnahmen, die den Blutdruck verringern, wirken sich positiv auf das Risiko für Herzinfarkt und Schlaganfall aus. Eine solche Maßnahme könnte Magnesium sein, dem eine günstige Wirkung auf die Hypertonie zugeschrieben wird - u. a. durch einen reduzierten peripheren Gefäßwiderstand oder die Freisetzung von Stickstoffmonoxid. Nun untersuchte eine Metaanalyse, ob sich eine Mg-Supplementation auf den Blutdruck von Patienten auswirkt.

Es gibt viele Studien über die Auswirkungen von Mg-Präparaten auf den Blutdruck - meist jedoch bei gesunden Probanden oder kombiniert mit Patienten mit verschiedenen chronischen Erkrankungen. Entsprechend breit streuen die Größenordnungen des beobachteten Effekts der Mg-Supplementation. Die vorliegende Metaanalyse schloss daher nur Studien ein, die den Einfluss von zusätzlichem Mg auf den Blutdruck oder die Hypertonie bei einer vorerkrankten Studienpopulation untersuchte. Im Einzelnen waren dies Patienten mit Insulinresistenz oder Prädiabetes und nicht übertragbaren chronischen Erkrankungen, wie z.B. Typ-2-Diabetes. Leider fanden sich keine Studien, die eine Mg-Supplementation bei Patienten mit Nieren- oder Tumorerkrankungen im Hinblick auf den Blutdruck untersuchten.

Alle eingeschlossenen Studien sollten randomisiert und gegen Placebo kontrolliert sein, die Werte für den systolischen und diastolischen Blutdruck zu Studienbeginn und -ende angegeben und als primären Endpunkt den Blutdruck untersucht haben. Dies traf auf 11 Studien mit insgesamt 543 Patienten zu. Die zugeführte Mg-Dosis in den einzelnen Studien

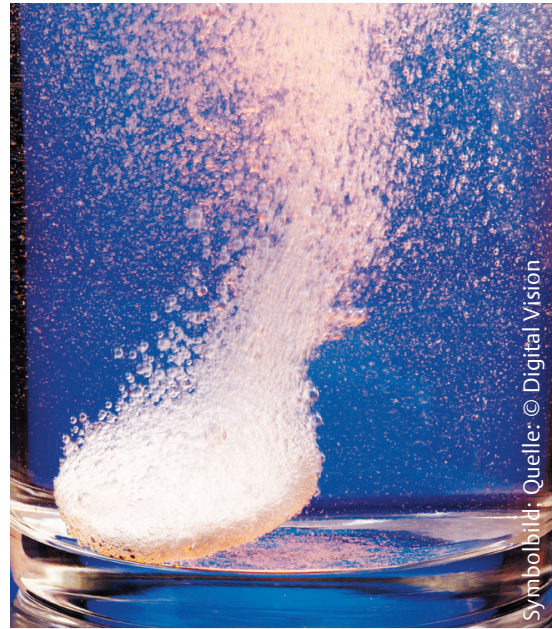

- Magnesium ist als essenzieller Mineralstoff für viele Stoffwechselvorgänge relevant. Bei Magnesiummangel kann die regelmäßige Einnahme von Magnesiumpräparaten blutdrucksenkend wirken.

reichte von 365-450 mg/Tag - in Form von Mg-Chlorid, Mg-Apartat, Mg-Pidolat oder einem speziellen Mg-Granulat. Die Studien dauerten im Follow-up 1-6 Monate (im Mittel 3,6 Monate).

Die gepoolten Analysen aus dem Random-Effects-Modell zeigen einen signifikanten Rückgang des Blutdrucks in der untersuchten Studienpopulation. Dies entspricht bei der Mg-Gruppe, verglichen mit der Kontrollgruppe, einem gewichteten mittleren Rückgang von

- 2,22 mmHg im systolischen Blutdruck und

- 2,54 mmHg im diastolischen Blutdruck.

Im Mittel verringerte die Mg-Supplementation beim Vergleich der Messungen zu Beginn und Ende der Studie den

- systolischen Blutdruck um 4,18 mmHg und

- den diastolischen Blutdruck um $2,27 \mathrm{mmHg}$.

Da etwa 60-80\% aller Diabetespatienten an kardiovaskulären Komplikationen versterben und knapp 75\% derartiger Komplikationen einem hohen Blutdruck zugeschrieben werden, ist selbst eine nur mäßige Senkung eines hohen Blutdrucks klinisch relevant - und dies trotz der heterogenen Patientengruppe in den eingeschlossenen Studien.
FAZIT

Gemäß dieser Metaanalyse wirkt Mg günstig auf den Blutdruck. Deshalb könnten nach Ansicht der Autoren Patienten mit Hypertonie und präklinischen metabolischen Erkrankungen wie Insulinresistenz, Prädiabetes, Typ-2-Diabetes oder kardiovaskulären Erkrankungen von einer MgSupplementation profitieren - und zwar sowohl direkt durch den gesenkten Blutdruck als auch indirekt über die gebesserte Stoffwechsellage ihrer Begleiterkrankungen, die ja ihrerseits für einen erhöhten Blutdruck prädisponieren.

Dipl.-Biol. Ulrike Andres, Ginsheim 